\title{
Low Cost Test Bench to Assess Energy Efficiency of Communications Network Equipment
}

\author{
Luiz Henrique Biazotto, \\ Alexandre de Assis Mota and Lia Toledo Moreira Mota \\ Exact, Environmental and Technological Sciences Center, \\ Pontifical Catholic University of Campinas, \\ Rodovia D. Pedro I, km 136, Parque das Universidades, \\ Campinas-SP, CEP 13086-900, Brazil
}

Received 2012-07-27, Revised 2012-09-13; Accepted 2012-09-18

\begin{abstract}
Communication network Quality of Service (QoS) degradation can lead to the consumption of additional energy to exchange information than that needed in normal conditions. If such behavior is known, network management can be more efficient and accurate, permitting to control more efficiently the energy consumption in telecommunication equipments. This study describes a test bench that can simultaneously measure the QoS parameters and energy consumption in telecommunication networks, in different traffic conditions and in different parts and components of a reduced communication network. The implemented test bench allows the accomplishment of different experiments. The QoS and power data can be presented as graphs that correlate them both. The results show that network quality of service degradation imply in more energy consumption compromising Energy Efficiency in the network.
\end{abstract}

Keywords: Electric Power, Quality of Service, Energy Consumption, Telecommunications Network, Energy Efficiency, Test Bench

\section{INTRODUCTION}

Currently, there is a strong concern in the world context, focusing on ensuring the energy provision of essential equipment, especially to those that compose the telecommunications network infrastructure. This concern is justified insofar as a breakdown in communication systems, mainly in the World Wide Web, the Internet, may lead to a chaotic situation of disinformation (Biazotto et al., 2011). On the other hand, the electrical energy consumption in Brazil in January 2010 showed an increase of $9.1 \%$ related to the same period of the year before, with all sectors presenting a significant increase of consumption. Given this situation, developers of telecommunication equipments are even more concerned with the excessive consumption of energy by telecommunication networks (Baliga et al., 2011; Gupta and Singh, 2003). Although isolated network equipment can represent an insignificant energy consumption related to the total demand of the system, the concatenated effect of all network equipments can show pretty high values of high energy consumption.

According to the research realized by GARTNER (Tully, 2010), the computer usage grows about $10 \%$ per year and also it was considered that in 2010 about 272 TWh were spent just with personal computers and 136 TWh with servers worldwide.

Several network features, for example, the noise, the packet loss, the retransmission of data, among other factors that may occur (Mota et al., 2010), can lead the equipment the consumption of more energy than needed to exchange information. It becomes interesting, therefore, to exploit the possibility of identifying the parameters that can be optimized in these equipments, aiming a lower energy consumption. Moreover, if such parameters are known, it's possible to make the network management more efficient and accurate, taking into account parameters of Quality of Service

Corresponding Author: Luiz Henrique Biazotto, Exact, Environmental and Technological Sciences Center, Pontifical Catholic University of Campinas, Rodovia D. Pedro I, km 136, Parque das Universidades, Campinas-SP, CEP 13086-900, Brazil 
(QoS), in order to detect and isolate failures that can increase the energy consumption, or even deploy devices that can control more efficiently the energy consumption in telecommunication equipments. Several algorithms can be used in this optimization, such as evolutionary approaches.

One can understand that Quality of Service (QoS) is a set of parameters which will influence the user's connection (Adibi et al., 2010). Some of these parameters can be:

- Bandwidth

- Latency

- Jitter

- Packet Loss

- Packet Delay

Depending on the behavior of the network, it's expected that it consumes more energy if there is signal degradation according to the parameters of QoS. It's possible to measure the energy utilization EI of equipment during a certain period of time as shown in equation 1 :

$$
E l=\int_{t_{1}}^{t_{2}} P(t) \cdot d t
$$

where,

$\mathrm{t}_{1}=$ is the initial time of measurement

$t_{2}=$ is the final time of measurement

$\mathrm{P}=$ is the power of the equipment, which is given by the instantaneous product of voltage and current

In this context, the main goal of this study is to describe a test bench that can measure the parameters of energy consumption in telecommunication networks, subjecting a network of reduced dimensions to different traffic conditions, in order to check the energy consumption in different parts and components of it, while monitoring the parameters of quality of service at the same time. The obtained results can be used to assess the energy efficiency of communications network equipment during communication processes. Also, the materials and software were selected in order to minimize costs and are easy to find, making feasible the test bench implementation with low investment in a wide range of situations.

\section{MATERIALS AND METHODS}

In the following, the hardware and software architecture of the implemented test bench are described. It was designed to be flexible, allowing to hold experiments that will enable the simultaneous measurement of QoS parameters and the equipment energy consumption during telecommunications processes.

This study, however, describes only the minimum necessary structure to measure the current and the voltage of the equipment involved. Other electrical variables could be measured, but that implies in hardware addition to the bench and, therefore, were not considered in this study.

\subsection{Hardware Architecture}

To set up the test bench it is necessary a workstation, a laptop and a wireless router, as below:

- Workstation Hardware configuration:

- Intel Pentium 4

- $\quad 512 \mathrm{MB}$ of RAM

- Motherboard with Intel chipset

- Network Card for $54 \mathrm{Mbps}$

- Monitor 15 “CRT

- Laptop Hardware Configuration:

- Intel Core 2 Duo T8100 2.10 GHz

- $4 \mathrm{~GB}$ of RAM

- Motherboard with Intel chipset

- $\quad$ Network Card for 54 Mbps

- Router Hardware Configuration:

- $\quad$ Model: TP-Link TL-WR741ND

- 150 Mbps Wireless N Router

- Dbi 5Antenna

- Power Meter Hardware Configuration

In this study, a microprocessed evaluation board of STMicroeletronics was adopted as the bench's power meter. This board, model STEVAL-ITE006V1, is able to perform the following measurements:

- Active Power $(\mathrm{KW})$ : Is the power that actually performs the study

- Reactive power (KVAR): Is used to create the electromagnetic field of inductive loads

- Apparent Power (KVA): Is the sum of active and reactive powers

- Active energy $(\mathrm{kWh})$ : Use of active power at a certain time interval

- Voltage: AC Voltage of the grid (127V or 220V)

- Current: RMS current consumed by the load

- Frequency: Frequency of the grid (in Brazil, the nominal frequency is $60 \mathrm{hz}$ ) 


\subsection{Software Architecture}

To set up the test bench different software packages are needed, as follows:

- Workstation Software Configuration:

- Microsoft Windows XP Professional with Service Pack 3

- High End Meter from STMicroelectronics

- DesktopSpyCamera (software used to remove a print from the computer screen with the information of energy consumption as measured by power meter)

- JPERF (Server)

- Laptop Software Configuration:

- Microsoft Windows 7 with Service Pack 1

- JPERF (Client)

- Wireshark

\subsection{Network Architecture}

The set up network architecture is described in Fig. 1. Basically, communication processes can be established between the desktop (as a server) and the laptop (as a client), passing through the router. JPERF software is used to deploy communication packets on the network in a supervised way, while both JPERF and Wireshark software monitor the traffic and capture the QoS parameters. The ST board measures electrical power consumption that, after mathematical treatment, can compose a data bank that allows to correlate QoS and Energy performance in the test network. The link among stations can be wireless or cabled; in Fig. 1, both computers are connected via IEEE802.11 wireless channel.

\section{RESULTS AND DISCUSSION}

The implemented test bench, shown in Fig. 2, allows the accomplishment of the following experiments:

- Measurement of the energy consumption in a regular situation, i.e., without transmitting data for the mapping of energy consumption of the involved equipment

- Use of JPERF to insert packets of data in the network and analyze how the energy consumption of the network equipment will be

- Use of Wireshark to check what happens in the network

- Increase in the volume of data to check the correlation with the energy consumption

- Alteration in the parameters of quality of service to check the network behavior related to the energy consumption

- Other possibilities can also be adjusted and implemented using the same test bench

\subsection{Examples of Quality of Service and Power Data Measurement}

The QoS and power data, obtained simultaneously with the test bench, can be presented as graphs that correlate the measurements. As examples, Fig. 3-5 show, respectively, the relation between power usage by the laptop and network throughput, total packets transmitted and bandwidth as capture by Wireshark, considering time window of $1 \mathrm{~min}$.

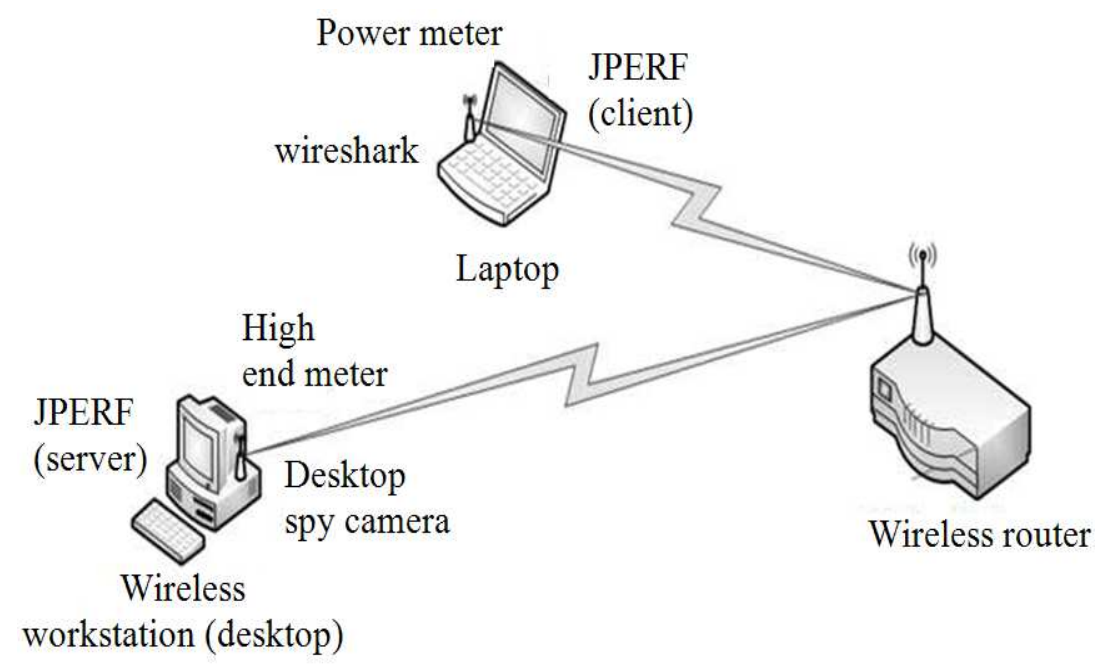

Fig. 1. Network architecture of the experiment 
Luiz Henrique Biazotto et al. / Journal of Computer Science 8 (11) (2012) 1849-1853

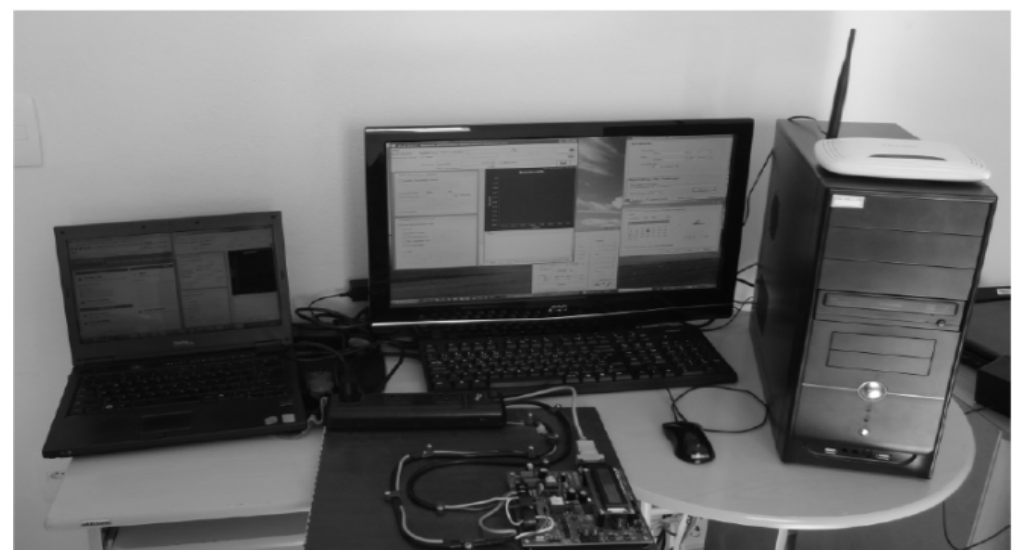

Fig. 2. Test bench

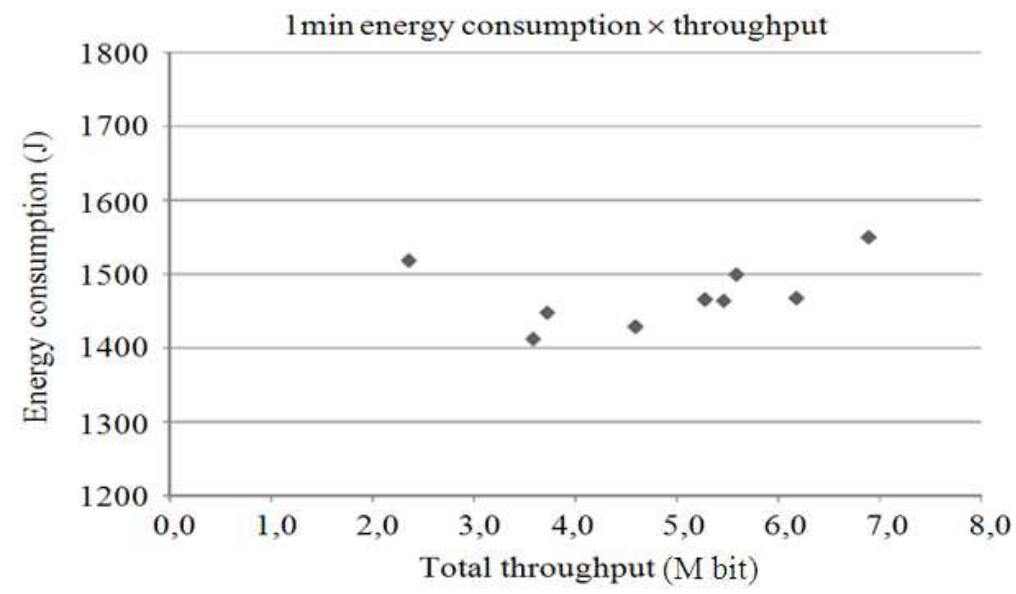

Fig. 3. Relation between the energy consumption versus throughput during $1 \mathrm{~min}$

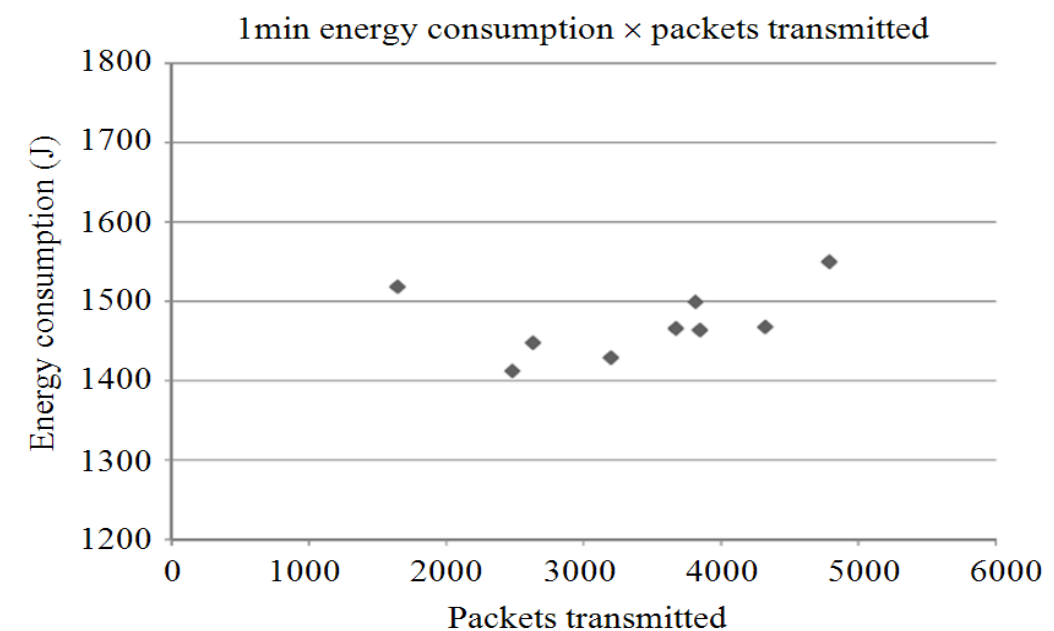

Fig. 4. Relation between the energy consumption versus transmitted packets in the network during 1 min 


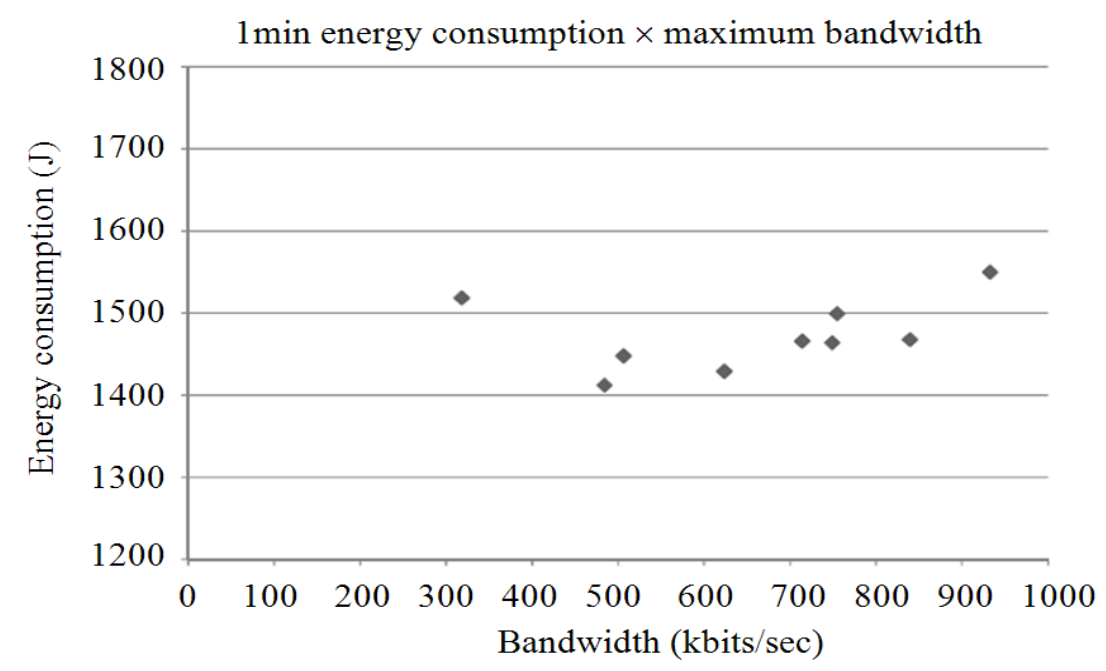

Fig. 5. Energy consumption versus bandwidth in the data link of the experiment, during $1 \mathrm{~min}$

\section{CONCLUSION}

As seen in Fig. 3-5, the data collected during the test bench's experiments can show the relation between the electric energy consumption and the parameters of Quality of Service (QoS) for a specific network component or station.

The graphs represented in Fig. 3-5 also show that the greater the effort exerted by the network to transmit information is, the greater will be the electric energy consumption in the network. It can be concluded, then, that network quality of service degradation imply in more energy required by its components (stations and link equipment).

\section{REFERENCES}

Adibi, S., R. Jain, S. Parekh and M. Tofighbakhsh, 2010. Quality of Service Architectures for Wireless Networks: Performance Metrics and Management. 1st Edn., IGI Global Snippet, Hershey, ISBN-10: 1615206809, pp: 691.

Baliga, J., M.Z. Feng, R.W.A. Ayre and R.S. Tucker, 2011. Power consumption and energy efficiency in the internet. IEEE Network, 25: 6-12. DOI: 10.1109/MNET.2011.5730522
Biazotto, L.H., A.J.F. Peris, F.S. Cyriaco, O.C. Branquinho and A.A. Mota et al., 2011. A mapping energy consumption method in telecommunications networks. Proceedings of XL IGIP-International Symposium of Engineering Education, (PISEE' 11), pp: 700-703.

Gupta, M. and S. Singh, 2003. Greening of the internet. Proceedings of the 2003 Conference on Applications, Technologies, Architectures and Protocols for Computer Communications, Aug. 2529, ACM Press, Karlsruhe, Germany, pp: 19-26. DOI: $10.1145 / 863955.863959$

Mota, L., A. Mota and R. Rondon, 2010. Analysis of indoor signal attenuation in wireless networks using fuzzy logic. Int. J. Comput. Sci. Network Sec., 10: $78-85$.

Tully, J., 2010. Powerful argument for cutting IT energy consumption. 